\title{
STABILIZATION OF NONLINEAR SYSTEMS BY SIMILARITY TRANSFORMATIONS
}

\author{
IRINA E. ZUBER \\ "Ecology" Shpalernaya St. 36 \\ St. Petersburg \\ Russia
}

(Received March, 1996; Revised November, 1997)

For a system $\dot{x}=A(x)+b(x) u, u(x)=s^{*}(x) x, \quad x \in \mathbb{R}^{n}$, where the pair $(A(x), b(x))$ is given, we obtain the feedback vector $s(x)$ to stabilize the corresponding closed loop system. For an arbitrarily chosen constant vector $g$, a sufficient condition of the existence and an explicit form of a similarity transformation $T(A(x), b(x), g)$ is established. The latter transforms matrix $A(x)$ into the Frobenius matrix, vector $b(x)$ into $g$, and an unknown feedback vector $s(x)$ into the first unit vector. The boundaries of $\tilde{A}(y, g)$ are determined by the boundaries of $\left\{\frac{\partial^{k} A(x)}{\partial x^{k}}, \frac{\partial^{k} b(x)}{\partial x^{k}}\right\}, k=$ $\overline{0, n-1}$. The stabilization of the transformed system is subject to the choice of the constant vector $g$.

Key words: Similarity Transformation, Derivative in Virtue of System, Stabilization.

AMS subject classifications: 34A99, 15A99, 15A04.

\section{Introduction}

Consider the system

$$
\dot{x}=A(x) x+b(x) u, \quad u(x)=s^{*}(x) x, \quad x \in \mathbb{R}^{n}
$$

where the pair $(A(x), b(x))$ is given and feedback vector $s(x)$ is to be determined. We intend to construct a similarity transformation matrix $T=T(A(x), b(x))$ that transforms matrix $A(x)$ into Frobenius matrix $\widetilde{A}(x)$ of the similar system

$$
\dot{y}=\tilde{A}(y) y+\tilde{b}(y) u, u(y)=\tilde{s}^{*}(y) y, \tilde{A}(y)=T(x) A(x) T^{-1}(x)+\dot{T}(x) T^{-1}(x) .
$$

A solution to the dual problem is known. For a given totally observable pair $(A(x), s(x))$, the similarity transformation is defined, that provides a Frobenius form for the matrix of the similar system and transforms the given vector $s(x)$ into $e_{1}=$ 
$(1,0, \ldots, 0)^{*}[1]$

The primal and dual problems are equivalent for linear systems. However, there is no such equivalence for nonlinear systems.

Furthermore, the solution to the primal problem is obtained for nonlinear systems without reference to dual analogs. The similarity transformation matrix $T=$ $T(A(x), b(x), g)$ is determined by the choice of a vector $g=$ const. The transformed system's stabilization is achieved by a proper choice of vector $g$.

\section{The Setting of the Problem}

We assume that the given pair $(A(x), b(x))$ is continuously differentiable $2 n-1$ times.

Our goal is to determine vector $s(x)$, which provides exponential stability of the closed loop system (1).

\section{Main Results}

We begin with the construction of a similarity transformation matrix, which provides the Frobenius form for the matrix of the transformed system. Consider the operators

$$
\begin{aligned}
& \mathcal{L}(x)=\ell_{1}(x)=\left[A(x)+\frac{d}{d t}\right] x \\
& \text { and } \quad \ell_{k}(x)=\mathscr{L}_{k-1}\left(\mathcal{L}_{1}(x)\right) .
\end{aligned}
$$

These operators determine the derivatives with respect to the system $\dot{x}=A(x) x$. They can be described by the corresponding matrix $L(x)$ as $\mathcal{L}(x)=L(x) x$ [1].

Consider the similarity transformation $y=T(x) x$, such that for an arbitrary $s(x)$ we have

$$
\begin{gathered}
y_{1}=s^{*}(x) x, \\
y_{2}=\frac{d}{d t} y_{1}, \\
\cdots \cdots \cdots \cdots \\
y_{n}=\frac{d}{d t} y_{n-1}=\frac{d^{n-1}}{(d t)^{n}} y_{1} .
\end{gathered}
$$

Here and further, if a derivation is performed with respect to the system $\dot{x}=A(x) x$, we have

$$
y_{k}=\frac{d^{k} s^{*}}{(d t)^{k}} x+c_{k}^{1} \frac{d^{k-1}}{(d t)^{k-1}} s^{*} L_{1} x+\ldots+c_{k}^{k} \frac{d s^{*}}{d t} L_{k-1} x .
$$

Then, according to [1], the matrix of the transformed system $\tilde{A}(y)=$ $T(x) A(x) T^{-1}(x)+\dot{T}(x) T^{-1}(x)$ is a Frobenius matrix with the last linearly independent row $\left(a_{1}(y), \ldots, a_{n}(y)\right)$ and an arbitrary (unknown) vector $s(x)$ turned into the unit vector $e_{1}=(1,0, \ldots, 0)^{*}$.

Consider matrix $T(x)$ defined by (4): 


$$
T(x)=\left|\begin{array}{c}
s^{*}(x) \\
\frac{d}{d t} s^{*}(x)+s^{*}(x) L_{1}(x) \\
\ldots \ldots \ldots \ldots \\
\sum_{j=0}^{n-1} c_{n-1}^{j} \frac{d^{j}}{(d t)^{j}} s^{*}(x) L_{n-1-j}(x)
\end{array}\right|
$$

with $t_{k}^{*}=\sum_{j=0}^{k-1} c_{k-1}^{j} \frac{d^{j}}{(d t)^{j}} s^{*}(x) L_{k-1-j}(x)$.

Consider an arbitrary vector $g=$ const and construct vector $\tilde{b}(y)=T(x) b(x)$ by equating $T(x) b(x)=g$. Then,

$$
g_{k}=\sum_{j=0}^{k-1} c_{k-1}^{j} \frac{d^{j}}{(d t)^{j}} s^{*}(x) L_{k-1-j}(x) b(x)=\mathrm{const}
$$

i.e.

$$
\frac{d^{m}}{(d t)^{m}} g_{k} \equiv 0, \quad \text { for } k, m=\overline{1, n}
$$

Then, $g_{1}^{(1)}=\frac{d}{d t} g_{1}=\frac{d}{d t} s^{*}(x) b(x)+s^{*}(x) \frac{d}{d t} b(x) \equiv 0$,

$$
g_{1}^{(k)}=\frac{d^{k}}{(d t)^{k}} g_{1}=\sum_{j=0}^{k} c_{k}^{j} \frac{d^{k-j}}{(d t)^{k-j}} s^{*}(x) \frac{d^{j}}{(d t)^{j}} b(x) \equiv 0 .
$$

Then, $g_{2}=g_{2}-g_{1}^{(1)}=s^{*}(x) f_{1}(x)$, where

$$
f_{1}(x)=\left(L_{1}(x)-\frac{d}{d t}\right) b(x) .
$$

then, $g_{2}^{(k)}=\frac{d^{k}}{(d t)^{k}} g_{2}=\sum_{j=0}^{k} c c_{k}^{j} \frac{d^{j}}{(d t)^{j}} s^{*}(x) \frac{d^{k-j}}{(d t)^{k-j}} f_{1}(x)$, and

$$
g_{3}=g_{3}-2 g_{2}^{(1)}-g_{1}^{(2)}=s^{*}(x)\left(f_{2}(x)-2 \frac{d}{d t} f_{1}(x)\right),
$$

where

$$
f_{2}(x)=\left(L_{2}(x)-\frac{d^{2}}{(d t)^{2}}\right) b(x) .
$$

Consider the vector sequence $r_{i}(x)$ :

$$
\begin{gathered}
r_{0}(x)=b(x), \quad r_{1}(x)=f_{1}(x) \\
r_{k}(x)=f_{k}(x)-\sum_{j=1}^{k-1} \overline{\bar{C}} j_{k} \frac{d^{j}}{(d t)^{j}} f_{k-j}(x),
\end{gathered}
$$

where

$$
f_{k}(x)=\left(L_{k}(x)-\frac{d^{k}}{(d t)^{k}}\right) b(x)
$$

Then, we have $g_{1}=s^{*}(x) r_{0}(x), g_{2}=s^{*}(x) r_{1}(x)$, and by induction we obtain the 
formula

$$
g_{k}=g_{k}-\sum_{j=1}^{k-2} c_{k-1}^{j} g_{k-1}^{(j)}=s^{*}(x) r_{k-1}(x) .
$$

So, $g=G^{*}(x) s(x)$, where

$$
\begin{gathered}
G^{*}=\left|\begin{array}{c}
r_{0}^{*}(x) \\
\ldots \ldots \ldots \\
r_{n-1}^{*}(x)
\end{array}\right| \\
s(x)=G^{-1 *}(x) g .
\end{gathered}
$$

The above results can be summarized as Theorem 1 .

Theorem 1: Assume that the matrix $G(x)$ in (9) is nonsingular for $x \in \mathbb{R}^{n}$ and the pair $\left(A(x), G^{-1 *}(x) g\right)$ is totally observable.

Then the similarity transformation matrix $T(x, g)$, defined by

$$
T=\left|\begin{array}{c}
g^{*} G^{-1}(x) \\
\frac{d}{d t} g^{*} G^{-1}(x)+g^{*} G^{-1}(x) L_{1}(x) \\
\ldots \ldots \ldots \ldots \ldots \\
\sum_{j=0}^{n-1} c_{n-1}^{j} \frac{d^{j}}{(d t)^{j}} g^{*} G^{-1}(x) L_{n-1-j}(x)
\end{array}\right|
$$

transforms the original matrix $A(x)$ into a Frobenius matrix $\tilde{A}(y, g)$, the vector $b(x)$ into $g$, and an arbitrary vector $s(x)$ into $e_{1}=(1,0, \ldots, 0)^{*}$.

Note now that, if system (1) is defined for $\|x\| \leq K$, we can estimate the norm of the corresponding matrix $\widetilde{A}(y, g)$. The last row of matrix $\tilde{A}(y, g)$ is defined by

$$
\frac{d y_{n}}{d t}=a_{n}^{*}(y, g) y=\frac{d}{d t} t_{n}^{*}(x) x+t_{n}^{*}(x) L_{1} x
$$

where $t_{n}^{*}(x, g)$ is the last row of matrix $T(x, g)$ defined by (11). Thus the elements of $a_{n}^{*}(y, g)$ depend linearly on the integers $g_{i}, i=\overline{1, n}$; i.e. they do not depend on $\|g\|$.

Replace vector $g$ in (11) by the unit vector $e_{i}$ and denote the obtained matrix of transformation by $T_{i}$ and the last row of the transformed system's matrix $\tilde{A}\left(y, e_{i}\right)$ by $a_{n}^{*}(y, i)=\left(\alpha_{i}(y, i), \ldots, \alpha_{n}(y, i)\right)$. If system (1) is defined for $\|x\|=K$, then there exists integers $m_{i}^{j}$ such that $\left|a_{i j}(y, i)\right| \leq m_{i}^{j}$ for $\|x\| \leq K, y=T_{i}(x) x$. Now consider an arbitrary constant vector $g \neq 0$ and construct matrix $T(x, g)$ defined by (11) and matrix $\widetilde{A}(y, g)$ of the system transformed by $y=T(x, g) x$. Then for elements of last row $\tilde{\tilde{a}}_{i}(y, g)=\left(\tilde{a}_{1}(y, g), \ldots, \tilde{a}_{n}(y, g)\right)$ of matrix $\tilde{A}(y, g)$, we obtain the estimate

$$
\left|\tilde{a}_{j}\left(y_{1}\right)\right| \leq m_{j}=\sum_{i} m_{i}^{j},\|x\| \leq K, y=T(x, g) x .
$$

Now considering a system similar to (1) by the transformation $T(x, g)$ we will select a vector $g=$ const which provides its stability and then we will return to (1) (if the inverse transformation exists). 
Consider the specified system:

where

$$
\dot{y}=A_{0}(y) y+r e_{1}^{*} y, \quad y \in Y \subset \mathbb{R}^{n},
$$

$$
A_{0}=\left|\begin{array}{ccccc}
0 & 1 & 0 & \ldots & 0 \\
0 & 0 & 1 & \ldots & 0 \\
\ldots & \ldots & \ldots \\
a_{1}(y) \ldots & \ldots & a_{n}(y)
\end{array}\right|, e_{i}=(1,0, \ldots, 0)^{*}, r=\text { const. }
$$

Consider a positive-definite matrix $H_{0}=P C P$, where $P=\operatorname{diag}\left\{h_{i}\right\}, h_{i}>0$, $i=\overline{1, n}, \quad c=\left\{c_{i j}\right\}, \quad c_{i i}=1, \quad c_{i i-1}=c_{i i+1}=-1 / 2, \quad c_{i j}=0, \quad j<i=1, \quad j<i+1$; according to [2] construct

$$
\begin{gathered}
r=\lambda H_{0}^{-1} e_{1}, \lambda=\mathrm{const} \\
V_{0}(y)=y^{*} H_{0} y .
\end{gathered}
$$

We intend to demonstrate that the proper choice of $n+1$ integers $h_{i}>0, i=\overline{1, n}$, $\lambda$, provides (for an arbitrary integer $\alpha>0$ ) the fulfillment of the condition

$$
D_{0}^{*}(y) H_{0}+H_{0} D_{0}(y)<-\alpha I
$$

where $D_{0}(y)=A_{0}(y)+r e_{1}^{*}$.

Let us introduce the following notation:

$$
\dot{V}_{0}(y)=y^{*} L(y) y=y^{*} Q_{0} y+y^{*}\left(e_{1} r^{*} H_{0}+H_{0} r e_{1}^{*}\right) y .
$$

Here $Q_{0}=A^{*} H_{0}+H_{0} A$ is the matrix of the derivative of form (15) in virtue of open loop system $\dot{y}=A_{0}(y) y$. Also set

$$
L_{\alpha}(y)=L(y)+\alpha I \quad Q_{\alpha}(y)=Q_{0}(y)+\alpha I \text {. }
$$

The negative definiteness of matrix $L_{\alpha}(y)$ is equivalent to the existence of $n$ sign changes in the sequence of the main diagonal minors of matrix $L_{\alpha}(y)$ (for every $y$ ) (Sylvester criterion [3]).

We begin the inspection of the diagonal minors from the right lower corner of the matrix.

Lemma 1: The first $n$ of the main diagonal minors of $L_{\alpha}(y), \Delta_{i}\left(L_{\alpha}\right), i=\overline{0, n-1}$, do not depend on $r$ and are therefore equal to the respective minors of matrix $Q_{\alpha}$.

The validity of the statement is a straightforward consequence of the structure of matrices $H_{0}$ and $A_{0}$ [2]. Now we propose to choose the parameters $h_{i}, i=\overline{1, n}$ as to guarantee $(n-1)$ sign changes in the $n$-tuple $\Delta_{j}, j=\overline{0, n-1}, \Delta_{0}=1$, of the main diagonal minors of matrix $Q_{\alpha}(y)$.

Lemma 2: Denote the right lower $(i \times i)$-corner of the matrix $Q_{\alpha}(y)$ by $Q_{\alpha}^{i}(y)$, its column by $q_{i}$, and its determinant by $\Delta_{i}$. Then the following assertions hold true:

1. The element $q_{i i}$ depends on $h_{n-j-1}$ linearly and does not depend on $h_{i}$ for $i<n-j-1$.

2. The element $q_{i j}$ does not depend on $h_{j}$ for $j<n-i$.

3. The required sign of $\Delta_{i}$ (and its consistency) may be reached throughout the choice of $h_{n-i-1}$.

The validity of the first two assertions follows directly from the structure of the matrices $A_{0}$ and $H_{0}$. The validity of Assertion 3 follows from the equality [3] 


$$
\Delta_{i}=\Delta_{i-1}\left(q_{i i}-q_{i}^{*}\left(Q_{\alpha}^{i-1}\right)^{-1} q_{i}\right) .
$$

Fixing $h_{n}\left(h_{n}=1\right.$, for example) we may now get the required $(n-1)$ sign changes in the sequence $\Delta_{j}\left(\Delta_{0}=1, \Delta_{1}, \ldots, \Delta_{n-1}\right)$ by the choice of integers $h_{n-1}, \ldots, h_{1}$ from the inequalities

$$
q_{n-1, n-1}-q_{1}^{*}\left(Q_{\alpha}^{i-1}\right)^{-1} q_{i}<0 .
$$

The choice of parameter $\lambda$ in (14) provides the fulfillment of the condition that $\operatorname{sign}\left(\operatorname{det} L_{\alpha}(y) / \Delta_{n-1} L_{\alpha}(y)\right)$ is negative. Indeed, in view of (14), $L_{\alpha}(y)=Q_{\alpha}+2 \lambda e_{1} e_{1}^{*}$ and consequently, $\operatorname{det} Q_{\alpha}+2 \lambda \Delta_{n-1}=\operatorname{det} L_{\alpha}$.

So, it is sufficient to choose

$$
\lambda \leq \sup _{y}\left|\operatorname{det} Q_{\alpha} / \Delta_{n-1}\right|
$$

to obtain the negative definiteness of $L_{\alpha}$. Thus, the following theorem is proved.

Theorem 2: For system (13) there exists, and is defined by the proper choice of integers $\lambda, h_{i}>0, i=\overline{1, n}$, vector $r$ described is formula (14), which provides exponential stability for the closed loop system (13)-(14) and the existence of its Lyapunov function (15).

Corollary: Assume that system (13) is defined for $\|y\| \leq K_{1}$. Then the domain of exponential stability for the closed loop system (13)-(14) is defined by the formula

$$
\|y\|^{2} \leq \frac{\lambda_{\min }\left(H_{0}\right)}{\lambda_{\max }\left(H_{0}\right)} K_{1}
$$

where $\lambda_{\min }\left(H_{0}\right)$ and $\lambda_{\max }\left(H_{0}\right)$ are minimal and maximal eigenvalues of the matrix $H_{0}$, respectively. The validity of this statement follows from Theorem 1.7 of [4].

Note: Consider system (1) for $\|x\| \leq K$ and choose vector $g$ as

$$
g=r
$$

where $r$ is defined according to Theorem 2 .

We assume that matrices $G(x)$ and $T(x)$ defined by formulas (9) and (11), respectively, are nonsingular for $\|x\| \leq K$. Then the transformation $y=T(x, g) x$ transfers closed loop system (1), where $s(x)=G^{-1 *}(x) g$, into system (13)-(14) for $\|y\| \leq K_{1}$, where

$$
K_{1}=\frac{K}{\operatorname{Sup}_{\|x\| \leq K} T^{-1}(x, g) \|} .
$$

In this case, the Lyapunov function of system (1) is determined by Lyapunov function (15) of system (13)-(14):

$$
\begin{gathered}
V(x)=x^{*} H(x) x, \\
H(x)+T^{-1^{*}}(x) H_{0} T^{-1}(x) .
\end{gathered}
$$

Now we stipulate for transformed system (2) the stabilizing vector $g=$ const given by Theorem 2 and return to the general type of system, i.e., to system (1). Then the following statement holds true.

Theorem 3: If matrices $G(x)$ and $T(x)$ defined by formulas (9) and (11) are nonsingular for $\|x\| \leq K$, then closed loop system (1), (11) is exponentially stable for

$$
\|x\| \leq \frac{\lambda_{\min }}{\lambda_{\max }} \frac{(H(x))}{(H(x))} K,
$$


and the Lyapunov function of this system is defined by equation (23).

The validity of this statement is obvious, because the nonsingularity of matrix $T(x)$ for $\|x\| \leq K$ provides the existence of the inverse transformation here. Note that nonsingularity of matrix $T(x)$ implies the total observability of the pair $\left(A(x), G^{*-1}(x) g\right)$.

To demonstrate the example, which follows, we must state explicitly what kind of matrix norm we intend to use. We choose the maximal eigenvalue of a matrix $\left(M^{*} M\right)^{1 / 2}$ as a norm of matrix $M$. Here the following lemma will be useful.

Lemma 3: Assume that $M$ is an arbitrary nonsingular matrix, $\operatorname{det} M \neq 0$. Then,

$$
\left.\left\|M^{-1}\right\| \leq S p\left(M^{*} M\right)\right)^{\frac{n}{2}}\left(\operatorname{det}\left(M^{*} M\right)\right)^{-\frac{n+1}{2 n}} .
$$

Here $S p(\cdot)$ is the sum of eigenvalues of $(\cdot)[3]$.

Proof of Lemma 3: Let $\pi=\left(S p\left(M^{*} M\right)\right)^{\frac{1}{2}}\left(\operatorname{det}\left(M^{*} M\right)\right)^{-\frac{1}{2 n}}$ and let $\mu(M)$ and $\nu(M)$ be maximal and minimal eigenvalues of the matrix $\left(M^{*} M\right)^{1 / 2}$, respectively.

Then $\mu\left(M^{-1}\right) / \nu\left(M^{-1}\right)=\mu(M) / \nu(M) \leq \pi^{n}[2]$. Hence, $\mu\left(M^{-1}\right) \leq$

$\pi^{n} \nu\left(M^{-1}\right) \leq \pi^{n}\left(\operatorname{det}\left(M^{*} M\right)\right)^{-2 / n}$, and, therefore, $\left\|M^{-1}\right\|=\mu\left(M^{-1}\right) \leq$

$\left(S p\left(M^{*} M\right)\right)^{\frac{1}{2}}\left(\operatorname{det}\left(M^{*} M\right)\right)^{-\frac{n+1}{2 n}}$.

\section{Example}

Consider the stabilization of system (1) for $n=4$. Then the matrix of system (13) is

$$
A(y)=\left|\begin{array}{cccc}
0 & 1 & 0 & 0 \\
0 & 0 & 1 & 0 \\
0 & 0 & 0 & 1 \\
a_{1}(y) & a_{2}(y) & a_{3}(y) & a_{4}(y)
\end{array}\right|,\left|a_{i}\right| \leq m_{i}, i=1,4 .
$$

We will construct matrix $H_{0}$ and vector $r=\lambda H_{0}^{-1} e_{1}$, which provide for arbitrary given $\alpha>0$ the fulfillment of the condition $\dot{V}<-\alpha V_{0}$. Form matrix $Q_{\alpha}=Q_{0}+\alpha I$,

\begin{tabular}{|c|c|c|c|c|}
\hline \multirow[t]{4}{*}{$Q_{\alpha}=\left\{q_{i j}\right\}_{i j=1}^{4}=$} & $2 \alpha h_{11}$ & $h_{11}+2 \alpha h_{12}$ & $a_{1} h_{34}+h_{12}$ & $a_{1} h_{44}$ \\
\hline & $h_{11}+2 \alpha h_{12}$ & $2\left(h_{12}+\alpha h_{22}\right)$ & $\begin{array}{l}h_{22}+2 \alpha h_{23} \\
+a_{2} h_{34}\end{array}$ & $a_{2} h_{44}+h_{23}$ \\
\hline & $a_{1} h_{34}+h_{12}$ & $\begin{array}{l}h_{22}+2 \alpha h_{23} \\
+a_{2} h_{34}\end{array}$ & $\begin{array}{l}2\left(h_{23}+\alpha h_{33}\right. \\
\left.+a_{3} h_{34}\right)\end{array}$ & $\begin{array}{l}\left(a_{4}+2 \alpha\right) h_{34} \\
+a_{3} h_{44}+h_{33}\end{array}$ \\
\hline & $a_{1} h_{44}$ & $a_{2} h_{44}+h_{23}$ & $\begin{array}{l}\left(a_{4}+2 \alpha\right) h_{34} \\
+a_{3} h_{44}+h_{33}\end{array}$ & $\begin{array}{l}2\left(h_{34}\right. \\
\left.+h_{44}\left(a_{4}+\alpha\right)\right)\end{array}$ \\
\hline
\end{tabular}
where $Q_{0}$ is the matrix of $\dot{V}_{0}$ with respect to open loop system (13), (25):

Here $h_{i j}=-h_{i} h_{j} / 2, h_{i i}=h_{i}^{2}$. Set $h_{4}=1$, and then from the condition $\Delta_{1}=q_{44}<-\epsilon / 2$, we obtain $h_{3}=\epsilon+m_{4}+\alpha$. Now consider $\Delta_{2}=q_{33} q_{44}-q_{34}^{2}$. The condition $\Delta_{2}>\epsilon / 2$ in view of (19) gives $h_{2} \geq h_{2}^{0}=h_{3}^{-1}\left(\operatorname{Sup}_{K}\left(q_{34}^{2}\left(h_{3}\right) / q_{44}\left(h_{3}\right)\right)+\right.$ $\left.\alpha h_{3}^{2}+m_{3}\right]$. 
Consider the next minor $\Delta_{3}\left(h_{3}, h_{2}, h_{1}\right)$. From the condition $\Delta_{3}<-\epsilon / 2$, we obtain, in terms of the notation in Lemma $2, h_{1}>\operatorname{Sup}_{\|y\| \leq K}\left(q_{3}^{*} Q_{3}^{-1} q_{3}\right)+\alpha h_{2}^{2}$, or in terms of Lemma $3, h_{i}=\epsilon+2 h_{2}^{2}+\operatorname{Sup}_{\|y\| \leq K}\left\|q_{3}\right\|^{2}\left(\operatorname{Sp}\left(Q_{3}^{*} Q_{3}\right)\right)^{\frac{n}{2}}\left(\operatorname{det}\left(Q_{3}^{*} Q_{3}\right)\right)^{-\frac{n+1}{2 n}}$. Matrix $H_{0}$ of form $V_{0}$ is therefore obtained. Now we find the integer $\lambda$ from the inequality (20), i.e., $\lambda<-\operatorname{Sup}_{\|y\| \leq K}\left|\operatorname{det} Q_{\alpha} / \Delta_{3}\right|$. Consider the similarity transformation $=T(x, g) x$ for the obtained vector $g=r$ and corresponding inverse transformation. Consider system (1) transformed from the stabilized system by the inverse transformation. Then vector $s(x)$, which provides exponential stability to system (1) for $n=4$, is defined by formulas $r=\lambda H_{0}^{-1} e_{1}, g=r, s(x)=G^{*-1}(x) g$, where $G(x)$ is given by $(9)$.

\section{Summary}

Stabilization of a class of smooth nonlinear systems is obtained. These results can be extended for time-varying systems.

The determined class of similarity transformation matrices $T(x, g)$ with an arbitrary constant vector $g$ provides the following form for the transformed system's matrix:

$$
\widetilde{D}(y, g)=\tilde{A}(y, g)+g e_{1}^{*}
$$

Here $\tilde{A}(y, g)$ is a Frobenius matrix: its norm does not depend on $g$ and for matrix $\tilde{A}(y, g)$, estimates (12) are valid. The obtained form for the closed loop system's matrix $\widetilde{D}(y, g)$ leads to optimization problems for system (1).

\section{Acknowledgement}

This work is supported by the Russian Foundation of Fundamental Research (project no. 95-00166a).

\section{References}

[1] Deza, F. and Gauthier, J.P., A simple and robust nonlinear estimator, Proc. of the 30th Conf. on Decision and Control, Brighton, England December (1991), 571-574.

[2] Dubrov, A.M. and Zuber, I.E., Design of exponentially stable control systems for a range of nonlinear processes, Automation and Remote Control 8 (1989), 33-40.

[3] Ganthmacher, F.R., The Theory of Matrices, Chelsea, New York 1969.

[4] Kuncevich, V.M. and Lichak, V.V., Synthesis of Automatic Control Systems by Using Lyapunov Functions, "Nauka", Moscow 1977. 


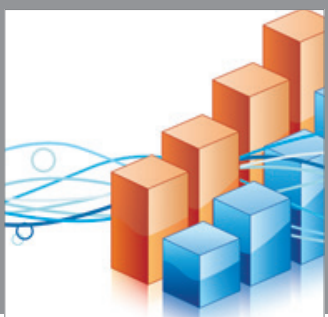

Advances in

Operations Research

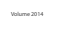

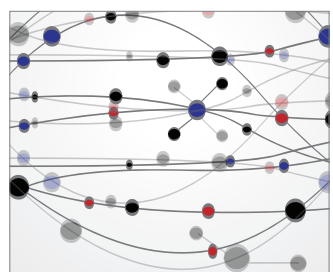

\section{The Scientific} World Journal
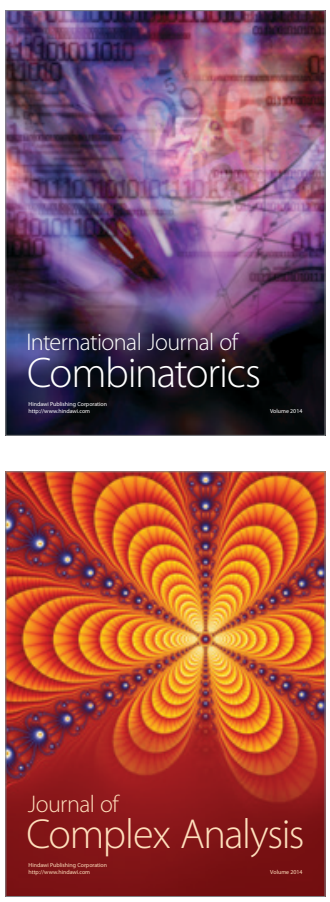

International Journal of

Mathematics and

Mathematical

Sciences
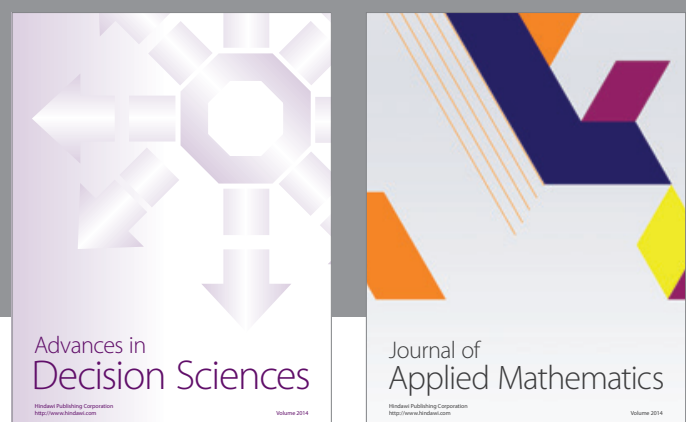

Journal of

Applied Mathematics
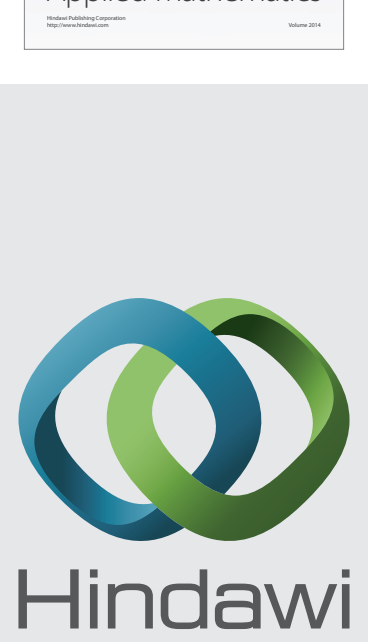

Submit your manuscripts at http://www.hindawi.com
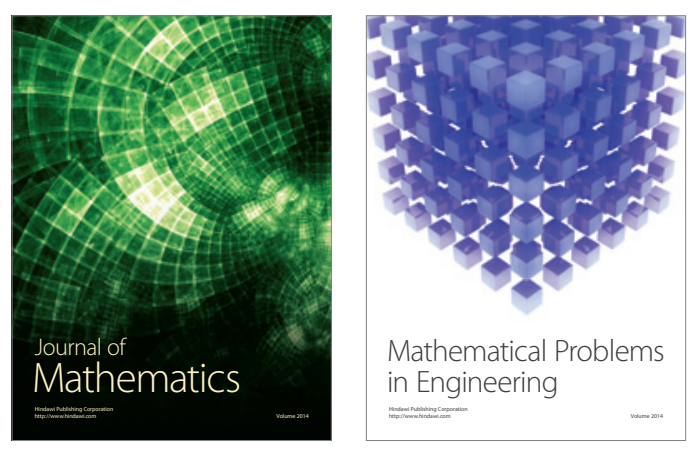

Mathematical Problems in Engineering
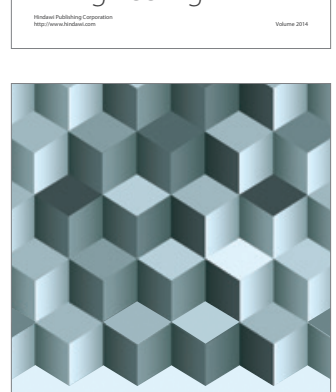

Journal of

Function Spaces
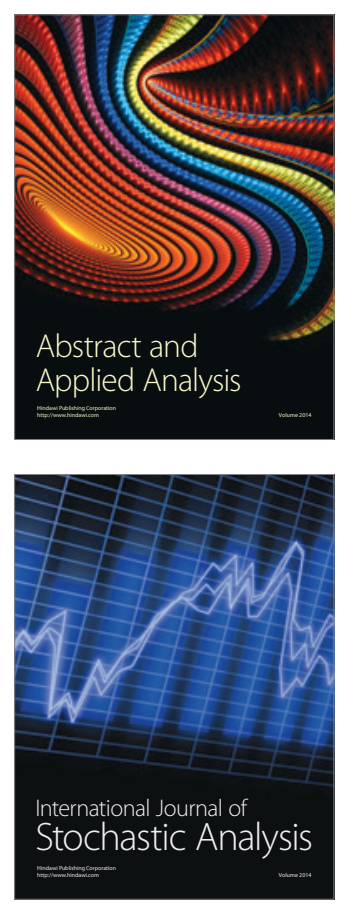

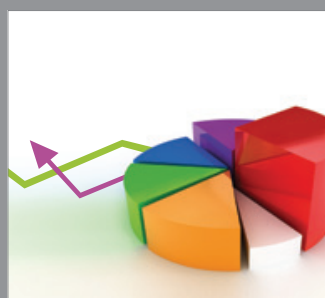

ournal of

Probability and Statistics

Promensencen
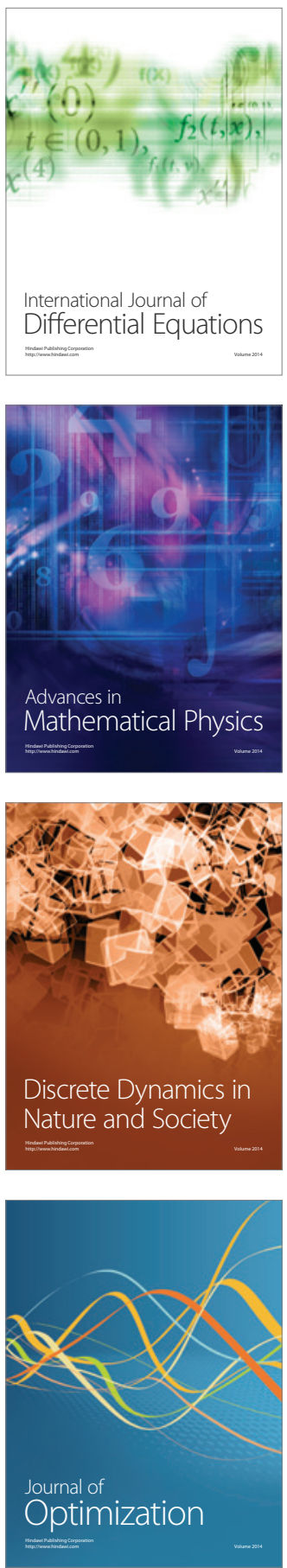